\title{
THERMO-FLUID DYNAMICS ANALYSIS AND AIR QUALITY FOR DIFFERENT VENTILATION PATTERNS IN AN OPERATING THEATRE
}

\author{
Carla Balocco ${ }^{1}$, Giuseppe Petrone ${ }^{2 *}$, Giuliano Cammarata ${ }^{2}$ \\ ${ }^{1}$ University of Firenze, Department of Industrial Engineering, Via S. Marta 3, 50139 Firenze, Italy \\ ${ }^{2}$ University of Catania, Department of Industrial Engineering, Viale A. Doria 6 - 95129 Catania, Italy
}

Contrary to other applications, the basic design criteria of a ventilation system and air distribution for operating rooms are not primarily those related to thermal comfort, but to indoor air quality(IAQ). The aim of the present research concerns the study of the performance of different ventilation schemes for a standard orthopaedic Operating Theatre (OT). Taking into account our previous studies of a real OT under operating use conditions, in this research different air flow configurations combined with some air curtain solutions on were investigated by numerical simulations. IAQ indexes and thermal comfort parameters, deduced from simulation results were calculated and discussed referring to the best performance and efficacy of the studied air flow schemes. Results comparison shows that the position in the center of the room and the large surface of the air inlet ceiling diffusers, compared to that of the air return grilles, guarantee the best efficacy of the air flow both for unidirectional vertical and horizontal. Moreover, some air curtain solutions on the ceiling allow the air distribution and quality control, limiting the effects of a shortcircuit between the air flow inlet and outlet and the contaminants reduction in critical zones.

Results obtained from CFD transient simulation applied to the two ventilation schemes studied are consistent with experimental data of recent literature. As a matter of fact, efficiency and efficacy of the unidirectional vertical air flow, improved with the air curtains application, are in good compliance with those reported in the recent literature. Our proposed ventilation schemes, equipped by unidirectional vertical downward air flow, comply with those pointed out by some important researches on this argument that demonstrate as this kind of ventilation system provides an ultraclean environment and the better comfort conditions for all the OT zones. Therefore, our investigation shows that the horizontal air flow system, can be an important alternative to the vertical one in the OT only if the supply and return diffusers are located on the opposite site of the same lateral wall and their position is chosen taking into account the location and height of the operating table, medical equipments, instruments tables and other obstructions, compared with the distance of the plenum and air inlet grilles: this result has been widely demonstrated by some authors with important findings concerning the most significant decrease of post-operative infection obtained with this ventilation solution. Comparison with recent researches on the ultra clean ventilation system performance against airborne infection in a real standard OT confirm our results validity. Implications of our findings highlight that ventilation scheme model closer to the center of the room and larger surface of the air supply diffusers connected with the position of the return grilles, the better the air flow performance is, when provided both by unidirectional vertical and horizontal air flows, especially if the use of air curtains mainly located at the ceiling is considered. High surface of the air supply diffusers combined with air curtains location to control the (both unidirectional vertical and horizontal) air flow direction and correct air recovery grille location, was identified as one of the most important factors in reducing short circuits between inlet and outlet air flows, guaranteeing the efficacy and efficiency of the ventilation system, in minimizing the thermal stratification effect, reducing contaminant concentration in the breathing and occupied/operating zone of the OT and also governing the dispersion of airborne infectious particles. 